\title{
Manejo de la angina crónica estable: Parte II. Tratamiento
}

El tratamiento de la angina crónica estable (ACE) tiene dos objetivos fundamentales:

1) Prevenir la aparición de eventos coronarios mayores (muerte súbita, infarto, angina inestable) y de insuficiencia cardíaca, que significativamente empeoran el pronóstico y aumentan la mortalidad.

2) Lograr que el paciente permanezca libre de síntomas y llegue a una capacidad funcional que no limite su calidad de vida, sobre la que debe hacerse una valoración individual.

El tratamiento de la ACE consiste en medidas generales y farmacológicas. Algunas tienen influencia sobre la sobrevida pero no sobre la angina, y otras pueden mejorar los síntomas con un efecto variable en la sobrevida.

Medidas para prevenir la mortalidad y la aparición infarto de miocardio. Medidas generales

Dados los avances tecnológicos en la enfermedad coronaria (EC), es habitual que los médicos tiendan a enfocar el manejo del paciente en el contexto de las intervenciones diagnósticas y terapéuticas, pasando por alto aspectos educativos y de calidad de vida del paciente. Enfocar estos aspectos permite trabajar más adecuadamente sobre los cambios en los hábitos y estilos de vida.

Es muy frecuente que una vez hecho el diagnóstico de EC el paciente desarrolle una sensación invalidez. Debe tenerse presente este aspecto para poder trabajar con él y su familia, mostrándole con claridad y simpleza que la adherencia al tratamiento y los cambios en el estilo de vida favorecen el pronóstico de la enfermedad. La historia natural de la ACE hace que la angina decline con el tiempo, como resultado del desarrollo de circulación colateral o por el efecto de programas de rehabilitación física. ${ }^{1}$

La frecuencia de los episodios de angina debe ser limitada al mínimo y debe contraindicarse cualquier actividad durante la cual se desarrollen los síntomas. El motivo para esta limitación es que la isquemia conduce a la hipomotilidad miocárdica y puede ocasionar arritmias, muerte súbita y un daño crónico sobre el corazón. Por esta razón debe indicarse al. paciente en forma preventiva que ante la aparición del angor debe detener completamente el esfuerzo y utilizar nitritos para que el episodio ceda más rápidamente.

Abandono del tabaco. Recomendación Clase I, nivel de evidencia B 2 Continuar fumando luego de un infarto está asociado a una mortalidad a los cinco años de un 50\% mayor con respecto a los que dejan de fumar; existe una relación lineal entre el riesgo de muerte cardiovascular y el número de cigarrillos consumidos. Este riesgo se pierde luego de 3 a 5 años de haber abandonado el hábito. La reducción de la mortalidad lograda por el abandono del tabaco es superior a la producida por los procedimientos de revascularización. ${ }^{3}$

Un metaanálisis* sobre tabaquismo pasivo en el domicilio o en el trabajo, mostró que también este está asociado con un aumento del riesgo de enfermedad cardiovascular del 20\%, por lo cual deberían implementarse medidas para evitarlo cuando está presente. ${ }^{4}$

Los pacientes con EC sintomática son el grupo más receptivo a los tratamientos para dejar de fumar. Alrededor de un $30 \%$ de los pacientes dejen de fumar luego del primer evento coronario, y este porcentaje puede ascender al $60 \%$ si se le adiciona un programa de cesación de tabaquismo. La rapidez y magnitud de la reducción del riesgo coronario luego de dejar de fumar hacen que esté presente en la mayoría de los programas de prevención secundaria de EC.

\section{Disminución del colesterol}

Disminución de colesterol en pacientes con ACE y LDL $130 \mathrm{mg} / \mathrm{dl}$. Recomendación Clase I, nivel de evidencia A 2

Disminución de colesterol en pacientes con ACE y LDL entre 100 y $130 \mathrm{mg} / \mathrm{dl}$. Recomendación Clase IIa, nivel de evidencia B2

En los últimos cuatro años ha surgido evidencia categórica sobre el beneficio del tratamiento de la hipercolesterolemia en los pacientes con EC. Las drogas utilizadas en la mayoría de los trabajos pertenecen al grupo de las estatinas que probablemente tengan un efecto beneficioso propio inhibiendo la activación de las placas de ateroma, independiente del descenso de la colesterolemia.
En el estudio escandinavo de sobrevida con estatinas (4S), se compararon dos grupos de pacientes hipercolesterolémicos con ACE con o sin infarto previo a tratamiento con simvastatina o placebo, que fueron seguidos por 5.3 años. ${ }^{5}$ El grupo tratado con simvastatina mostró una disminución de la mortalidad total del $30 \%$ con una reducción de los eventos coronarios fatales del $42 \%$. De acuerdo a este estudio se deberían tratar 30 pacientes hipercolesterolémicos durante 5 años para prevenir una muerte.

El estudio CARE (Cholesterol And Recurrent Events), incluyó pacientes post infarto con valores de colesterolemia dentro de los parámetros considerados normales. ${ }^{6}$ Estos pacientes fueron medicados con pravastatina a un promedio de $40 \mathrm{mg} /$ día ó un placebo idéntico y fueron seguidos por 5 años. Este estudio mostró una disminución del $24 \%$ en el resultado combinado de muerte súbita e infarto fatal y no fatal. De acuerdo a este estudio deberían tratarse 33 pacientes con colesterol normal y antecedentes de infarto durante 5 años para prevenir un nuevo infarto o una muerte por enfermedad coronaria.

En el estudio LIPID fueron seguidos durante 6 años 9014 pacientes medicados con $40 \mathrm{mg}$ de pravastatina contra placebo. ${ }^{7}$ Este estudio mostró que el tratamiento redujo en un $24 \%$ la mortalidad total y en un $24 \%$ en la mortalidad coronaria, el beneficio alcanzó a todos excepto a aquellos con un LDL inicial menor de $130 \mathrm{mg} \%$.

Un estudio reciente, siguió durante cinco años a 2531 hombres con EC, con un LDL menor a $140 \mathrm{mgdl}$ y un HDL menor de $40 \mathrm{mgdl}$, a los que randomizó a $1400 \mathrm{mg}$. de gemfibrozil y placebo. ${ }^{8}$ Se observó un aumento del $\mathrm{HDL}$ del $6 \%$ y de los trig licéridos del $31 \%$ y una reducción del riesgo de EC del $22 \%$ en el grupo tratamiento.

Se necesitarían tratar 25 pacientes de estas características durante 5 años para prevenir una muerte cardiaca o un infarto no fatal.

En todos los pacientes coronarios se debe investigar el perfil lipídico, ya que su reducción produce un importante impacto sobre a reducción de la mortalidad. Si éste es elevado el paciente debe recibir tratamiento indefinido con hipolipemiantes.

\section{Uso de agentes antiagregantes}

Recomendación Clase I, nivel de evidencia A. Uso de aspirina en ausencia de contraindicaciones ${ }^{2}$

Recomendación Clase IIa, nivel de evidencia B. Uso de ticlopidina o clopidrogel cuando la aspirina está absolutamente contraindicada ${ }^{2}$ La evidencia es concluyente con respecto a la disminución de la mortalidad que el ácido acetil salicílico (Aspirina) produce en los pacientes con enfermedad coronaria tanto aguda como crónica. Se ha demostrado una reducción de la mortalidad del $12 \%$ en pacientes tratados con antecedentes de infarto y del $32 \%$ en el riesgo de infarto en pacientes con angina crónica. ${ }^{9-10}$ El mecanismo por el cual reducen el riesgo de accidentes vasculares trombóticos es una inhibición en el sitio activo de la ciclooxigenasa, el cual se requiere para la producción de tromboxano A2, un potente promotor de la agregación plaquetaria. Existe con el uso de aspirina un riesgo de sangrado, complicaciones gastrointestinales y efectos con su uso crónico a nivel renal. El beneficio clínico de la aspirina ha sido demostrado en un rango de 75 a $1500 \mathrm{mg}$ diarios, pero las dosis altas no son más eficaces, excepto a nivel cerebral, y son probadamente más tóxicas. Todo paciente que no tenga contraindicaciones debe recibir entre 75 a $325 \mathrm{mg}$ diarios de aspirina indefinidamente.

En caso de alergia o contraindicación, puede utilizarse ticlopidina o clopidrogel, son in hibidores selectivos de la actividad plaquetaria mediante un bloqueo del adenosindifosfato plaquetario (ADP), también actúan a nivel de la interacción del fibrinógeno y el receptor IIB IIIA de membrana plaquetaria. La ticlopidina ocasiona neutropenia, este efecto adverso alcanza al 2,4\% de aquellos que utilizan esta droga dentro de los primeros 4 meses. Por este motivo su uso se ha limitado mucho debido a que el clopidrogel no posee este efecto adverso. Recientemente se ha reportado la presencia de púrpura trombocitopénica con esta droga, que es poco frecuente y aparece dentro de los primeros días de comenzado el tratamiento, pero al ser una droga nueva debemos estar atentos a la aparición de nuevos efectos adversos. ${ }^{11}$

Se estima que deberían tratarse con aspirina a 83 pacientes con enferme- 
dad coronaria durante 2.3 años para prevenir una muerte..$^{9}$ Debido al bajo costo de la droga y al beneficio de su uso, esta es una maniobra muy costo efectiva.

\section{Inhibidores de la enzima convertidora de Angiotensina Recomendación Clase I, nivel de evidencia A. Pacientes con fracción de eyección menor o igual al $40 \%$.}

La evidencia disponible muestra que aquellos pacientes con insuficiencia cardíaca posterior a un infarto o con unia fracción de eyección menor al $40 \%$, se benefician con la administración indefinida de inhibidores de la enzima convertidora de angiotensina (IECA). ${ }^{12}$ Se recomienda utilizar las dosis máximas según la tolerancia del paciente. Se ha estimado que se deben tratar entre 18 a 24 pacientes por 4 a 5 años después de un infarto con una función ventricular inferior al $40 \%$ con IECA para prevenir una muerte. ${ }^{13}$

\section{Terapia de reemplazo hormonal}

\section{Recomendación Clase IIb, nivel de evidencia B2}

Los resultados de estudios observacionales que evaluaron el uso de estrógenos solos o combinados con progesterona en prevención primaria, mostraron una reducción de la mortalidad cardiovascular de entre el 50 al $60 \%$. Pese a tratarse de estudios con seguimiento prolongado de gran cantidad de pacientes, al ser observacionales y no poder randomizar la intervención, tenían el riesgo de seleccionar mujeres más sanas y con mayor preocupación por la salud.

El único estudio randomizado de terapia de reemplazo hormonal (TRH) controlado con placebo, cuestiona profundamente los resultados de estos estudios. ${ }^{14}$ Este se realizó en mujeres con EC y no encontró diferencias a favor de los estrógenos. El grupo de pacientes que recibió TRH tuvo inicialmente una mortalidad mayor, adjudicada a un efecto procoagulante, que luego revirtió con una tendencia favorable, siendo nulo el resultado final. Con la evidencia actual su utlización como prevención en de EC en las mujeres no está establecida.

\section{Programas de rehabilitación}

Ejercicio físico programado. Recomendación Clase I, nivel de evidencia B2 Identificación y tratamiento de la depresión. Recomendación Clase II b, nivel de evidencia C2

Los programas de rehabilitación aportan a los pacientes un instrumento que les permite un mejor manejo de su enfermedad, accediendo a la educación médica sobre la patología y mejorando la adherencia a las medidas preventivas y a los cambios en el estilo de vida.

Todos los programas de rehabilitación tienen grupos de ejercicio físico controlado, cuyo beneficio ha sido bien documentado (mejora la capacidad funcional y tiene un efecto positivo sobre el perfil lipídico, la agregación plaquetaria y el sistema fibrinolítico). Algunos programas suelen contar con grupos para cesación del tabaquismo y apoyo psicológico.

Si bien no todos los programas son iguales ni funcionan de la misma manera, los pacientes que aceptan concurrir a los mismos, tienen un mejor pronóstico, ya que suelen ser adherentes y estar preocupados por su salud. Un metaanálisis* de los resultados globales de estos programas muestran una reducción significativa de la mortalidad total y cardiovascular del 20 al 25\%. ${ }^{15}$

\section{Tratamiento Médico para reducir la isquemia y mejorar la angina} La drogas antianginosas tienen un papel fundamental en el paciente con angina de pecho ya que permiten aliviar el dolor y prevenir su aparición a través de diferentes mecanismos de acción.

La efectividad de cada una de ellas depende de la causa subyacente de la isquemia.

\section{Nitritos}

Uso de NTG para el alivio inmediato de la angina. Recomendación clase I, nivel de evidencia C2

Son los fármacos más antiguos y esenciales utilizados para el control de la angina. Disminuyen el volumen intraventricular y la tensión en las paredes ventriculares reduciendo la precarga mediante venodilatación. Como consecuencia de estos cambios, desciende la demanda de oxígeno y aumenta el flujo arterial subendocárdico, mejorando la irrigación mio- cárdica. Los nitritos se encuentran disponibles como preparados de acción corta o en formulaciones de liberación prolongada.

Los preparados de acción corta permiten aliviar el angor durante el episodio agudo o evitar preventivamente la aparición del dolor ante una actividad que habitualmente lo desencadena. Entre ellos se encuentran el dinitrato de isosorbide (DNI) que se utiliza por vía sublingual y la nitroglicerina (NTG) que puede administrarse por vía sublingual o en aerosol. La NTG posee un tiempo de acción más rápido ante el comienzo de un dolor anginoso por lo cual resulta más conveniente para los ataques agudos y el DNI tiene un comienzo de ación más tardío pero más duradero por lo cual sirve para prevenir dolores previsibles. ${ }^{16}$

Es importante instruir al paciente para que use correctamente la medicación: en el momento de aparición del dolor debe detener totalmente la actividad que lo desencadenó, sentarse (para evitar la aparición de hipotensión ortostática sintomática, secundaria al efecto de la droga) y tomar una dosis de nitroglicerina o DNI sublingual. El comienzo de acción es casi inmediato (dos a tres minutos). Si el dolor persiste, puede repetir la dosis dos veces más (en total tres veces) con intervalos de tres a cinco minutos. Debe recomendársele que consulte rápidamente si el dolor no alivia ya que en ese caso podría estar cursando un infarto. Cuando estas formulaciones son utilizadas para evitar dolores previsibles, el procedimiento es similar.

En los pacientes con angina crónica estable se usan preparados de acción prolongada con el objeto de disminuir la frecuencia de los ataques. Las drogas disponibles son DNI administrado por vía oral, nitroglicerina indicada en forma de parches de acción prolongada o mononitrato de isosorbide (MNI) utilizado por vía oral.

Un problema frecuente con el uso de nitritos es que la exposición prolongada a estas drogas induce al desarrollo de tolerancia, es decir que el paciente necesita progresivamente dosis mayores para lograr el mismo efecto. La utilización de regímenes de prescripción que conserven un intervalo libre de droga (10 a 12 hs.), permite evitar este fenómeno. Esto puede lograrse indicando $20 \mathrm{mg}$ de DNI a las ocho de la mañana, a las doce del mediodía y a las cinco de la tarde o MNI a las siete de la mañana y a las dos de la tarde o parches de NTG con suspensión nocturna.

\section{Bloqueantes Beta}

Uso de beta bloqueantes como tratamiento inicial en pacientes con ACE e infarto previo. Recomendación Clase I, nivel de evidencia A2 Uso de beta bloqueantes como tratamiento inicial en pacientes con ACE sin infarto previo. Recomendación Clase I, nivel de evidencia B2 Si bien los nitritos son muy eficaces para el tratamiento del angor y mejoran la tolerancia al ejercicio, se desconoce si modifican el pronóstico del paciente a largo plazo. Los b bloqueantes son las únicas drogas antianginosas que han demostrado mejorar la sobrevida y reducir el riesgo de reinfarto en los pacientes con enfermedad coronaria. Estas drogas disminuyen la frecuencia de episodios anginosos y aumentan el umbral de la angina en los pacientes con angina crónica estable, reducen en un $40 \%$ la tasa de muerte súbita en los pacientes sobrevivientes de un infarto y en un $20 \%$ la mortalidad global. ${ }^{17-18}$

Los b bloqueantes actúan sobre los receptores b adrenérgicos produciendo una disminución de la demanda miocárdica de oxígeno como consecuencia de la reducción de la frecuencia cardíaca y la contractilidad (efecto cronotrópico e inotrópico negativo). La prolongación de la diástole mejora la perfusión miocárdica.

Los efectos beneficiosos sobre la mortalidad cardiovascular son producidos por cualquier tipo de b bloqueante excepto por aquellos que tienen actividad agonista adrenérgica intrínseca como el carvedilol, por este motivo no deben utilizarse en la prevención de enfermedad coronaria después de un infarto o como antianginosos. Si bien pueden utilizarse cualquiera de los b bloqueantes disponibles, los más convenientes son el atenolol y el metoprolol debido a su mayor cardioselectividad y menores efectos adversos. El atenolol tiene una vida media más prolongada y permite una posología más cómoda (una vez por día, dosis usual 25-100 mg/día). 
Para decidir la dosis óptima para cada paciente debe monitorearse la respuesta de su frecuencia cardíaca. La meta es lograr una frecuencia cardíaca basal de entre 50 y 60 latidos que no ascienda más del $25 \%$ durante un ejercicio leve ni más del 50\% durante un ejercicio intenso.

Debido a su efecto negativo sobre el crono e inotropismo, estas drogas están contraindicadas en los pacientes con bloqueo aurículoventricular, bradicardia sintomática y disfunción ventricular severa. Tradicionalmente se creía que los pacientes con insuficiencia cardíaca, infarto y fracción de eyección menor a 40\%, podían deteriorase con el uso de betabloquentes. Sin embargo en aquellos ensayos en que fueron incluidos este grupo de pacientes de riesgo, si eran bien tolerados, los beneficios sobre la mortalidad fueron muy importantes. Por lo cual sólo se debería excluir a aquellos que no los toleran. Se recomienda utilizarlos con cuidado en los pacientes asmáticos o con enfermedad pulmonar obstructiva crónica. La dosis debe titularse en forma gradual, empezando con dosis bajas que se aumentará cada dos semanas según respuesta y tolerancia clínica.

\section{Bloqueantes Cálcicos}

Los bloqueantes cálcicos representan un grupo heterogéneo de fármacos que se caracterizan por inhibir el transporte de calcio en los canales lentos de las membranas celulares de los vasos miocárdicos y otros tejidos de músculo liso. Como consecuencia de este efecto, producen una vasodilatación arterial que mejora la perfusión miocárdica optimizando la circulación colateral y redistribuyéndola hacia zonas afectadas por isquemia.

Los distintos bloquentes cálcicos difieren en su potencia sobre la vasodilatación arterial, acción inotrópica negativa e influencia sobre el sistema de conducción.

La nifedipina es el vasodilatador arterial más potente dentro de este grupo, con pocos efectos sobre la función ventricular izquierda y el sistema de conducción. Su potente efecto en la reducción de la poscarga tiende a incrementar la frecuencia cardiaca en forma refleja, por lo cual el consumo de oxígeno puede no disminuir. Esto ocasiona en algunos casos agravamiento de la angina. En estudios caso control* y en un metaanálisis* de uso de drogas en pacientes con ACE, la nifedipina se asoció con un incremento del riesgo de infarto de miocardio. ${ }^{19}$

El verapamilo es de estas drogas la que tiene mayor efecto sobre el sistema de conducción, también tiene efecto sobre la contractilidad. Su eficacia como antianginoso está probada en estudios que lo compararon con betabloquentes.

El diltiazem es uno de los bloqueantes cálcicos mejor tolerados. Comparado con verapamilo causa menor disminución sobre la actividad del nódulo sinusal y el nódulo AV, aunque está contraindicado en enfermedad del nódulo y bloqueo AV. En la angina estable producen disminución del número de episodios y aumento de la tolerancia al esfuerzo. La dosis de diltiazem es de 120 a $360 \mathrm{mg}$. día repartidas en cuatro tomas. Hay cápsulas de acción prolongada que su pueden utilizar en una a dos tomas diarias.

En estudios de pacientes post-infarto, tanto el verapamilo como el diltiazem demostraron reducir las tasas de reinfarto en pacientes con función ventricular normal; en cambio en pacientes con disfunción su efecto es adverso. ${ }^{20}$ No hay todavía estudios de sobrevida en pacientes con angina crónica sin infarto previo.

La amlodipina tiene una acción prolongada y tiene menos efecto inotrópico negativo. En pacientes con angina crónica, ha demostrado ser eficaz y bien tolerada.

Aunque los bloqueantes cálcicos no deben utilizarse como antisquémicos de primera línea, estos cumplen un rol importante en aquellos pacientes con angina que tienen un componente de vasoespasmo. En el caso de no poder utilizar beta bloqueantes, se recomienda utilizar diltiazem o verapamilo dado su efecto inotrópico y cronotrópico negativo. La amlodipina puede utilizarse si es necesario asociar un bloqueante cálcico a un beta bloqueante. La nifedipina debe evitarse, ya que por su mecanismo de acción, tiende a aumentar la isquemia.

\section{Régimen terapéutico combinado}

Si el paciente con ACE presenta intolerancia a a lgunas de las drogas o la angina no logra controlarse adecuadamente, está indicado combinar las drogas antianginosas. Si aun así la angina no se controla, debe pensarse en utilizar procedimientos de revascularización.

La droga de primera elección son los b bloqueantes. Cuando el paciente persiste sintomático a pesar del uso de b bloqueantes a dosis óptimas, y luego de verificar que su adherencia al tratamiento es buena, se plantea la necesidad de asociar otras drogas antianginosas al régimen terapéutico.

\section{a) b bloqueantes y nitritos}

Uso nitritos de accion prolongada asociado a beta bloqueantes cuando el tratamiento inicial con estos últimos no es exitoso. Recomendación Clase I, nivel de evidencia B

Esta combinación es sumamente útil ya que los efectos de ambas drogas se complementan. Los nitritos causan vasodilatación disminuyendo la precarga, mejorando la perfusión miocárdica, y los b bloqueantes disminuyen la frecuencia y la contractilidad cardíacas reduciendo la demanda de oxígeno. Además, el efecto cronotrópico negativo de los b bloqueantes compensa el incremento en la frecuencia cardíaca que ocasiona la vasodilatación provocada por los nitritos. Al mismo tiempo, estos últimos reducen la precarga y de este modo minimizan cualquier aumento de la presión de fin de diástole que pueda ocasionar el efecto inotrópico negativo de los b bloqueantes. ${ }^{21}$ Otro efecto beneficioso de esta combinación es la protección que brindan b bloqueantes en los períodos libres de nitritos.

\section{b) b bloqueantes y bloqueantes cálcicos}

En la práctica clínica los resultados de esta combinación son poco convincentes. El efecto negativo que ambas drogas tienen sobre la función ventricular y la conducción auriculoventricular es aditivo: ambos agentes son inotrópicos negativos y pueden empeorar la disfunción miocárdica en los pacientes con trastornos de la conducción o aumentar el riesgo de desarrollar un bloqueo auriculoventricular y bradiarritmias sintomáticas. La opción que minimiza estos efectos adversos es utilizar bloqueantes cálcicos con menor efecto sobre el sistema de conducción como la amlodipina.

\section{c) b bloqueantes, nitritos y bloqueantes cálcicos}

Uso de bloqueantes cálcicos, nitritos y beta bloqueantes cuando el tratamiento inicial con estos últimos no es exitoso. Recomendación Clase $\mathrm{I}$, nivel de evidencia B

La combinación de estas tres drogas se utiliza con frecuencia en los pacientes con angina crónica refractaria. Sus mecanismos de acción son lo suficientemente diferentes como para pensar que esta combinación sería útil. Sin embargo no hay ningún estudio controlado que haya evaluado esta combinación y brinde evidencia categórica para su uso. 21

\section{Procedimientos de revascularizacion}

Los procedimientos de revascularización son la angioplastia transluminal percutanea y la cirugía de puente coronario (by pass). Toda vez que se indica una coronariografía (CCG), es habitualmente con el criterio de encontrar lesiones de las arterias coronarias que sean plausibles de tratarse con procedimientos de revascularización. Posteriormente de acuerdo al tipo de lesiones, el cardiólogo decidirá que tipo de procedimiento es más adecuado para el paciente.

\section{a) Angioplastía}

La angioplastía consiste en la dilatación de la luz arterial estenosada mediante la colocación de un catéter balón. Si bien este procedimiento permite mejorar la circulación coronaria evitando la anestesia general, la toracotomía y la circulación extracorpórea, la angioplastía no es un procedimiento exento de riesgos. Entre las complicaciones inmediatas se encuentran la oclusión arterial aguda, la ruptura o disección de la arteria y la embolización distal. Tiene una mortalidad del $0.4 \%$ y entre el 2 y el $4 \%$ de los pacientes requieren cirugía de urgencia. En todo lugar que se realiza angioplastía debe existir un apoyo de un equipo de cirugía cardiovascular. 
El $90 \%$ de las lesiones se pueden dilatar satisfactoriamente pero entre un 20 y un $40 \%$ sufren reestenosis en el curso de un año y esta es la gran limitante del procedimiento.

Para intentar resolver el problema de la reestenosis luego de la angioplastía se desarrollaron dispositivos diseñados para mantener la luz de la arteria denominados stents "STENTS". Estos dispositivos consisten en finísimas mallas metálicas que se insertan en la arteria luego de la dilatación con el objeto de mantener una permeabilidad arterial adecuada. La decisión de colocar un STENT corresponde al especialista y depende de la localización y el tipo de lesión en la cual la reestenosis es más probable.

\section{Angioplastia vs. Tratamiento medico}

El estudio ACME, fue el primer estudio que comparo angioplastia con tratamiento medico, fueron randomizados 328 pacientes. El $64 \%$ de los pacientes del grupo tratado con angioplastia y el $46 \%$ del grupo con tratamiento médico estaba libre de síntomas de angina a los seis meses $(p<0,01),{ }^{22}$ Dieciseis de los 105 pacientes en el grupo angioplastía requirieron repetir este procedimiento y siete debieron ser operados. En el grupo con tratamiento médico once debieron hacerse un procedimiento de angioplastía, ninguno requirió cirugía. No se observaron diferencias en eventos coronarios luego de cinco años de seguimiento.

El estudio RITA-2 incluyó 1018 pacientes con ACE que tenían indicación de CCG al que los cardiólogos en forma ciega consideraron podían tratarse por igual con tratamiento médico ó con angioplastía, se randomizaron las dos intervenciones. ${ }^{23}$ Luego de 2,7 años de seguimiento, el $6,3 \%$ de los pacientes asignados a angioplastía sufrieron el resultado combinado de infarto ó muerte vs. el 3,3\% de grupo con tratamiento médico $(p=0,02)$. La mejoría de la angina de pecho fue mayor en aquellos tratados con angioplastía especialmente los que padecían inicialmente angina más severa.

\section{Indicaciones}

Las indicaciones de angioplastía son la presencia de lesiones significativas proximales, de uno o dos vasos en pacientes sintomáticos a pesar del tratamiento médico. Si el paciente posee enfermedad de un vaso ó dos vasos y responde adecuadamente al tratamiento médico, debe seguirse clínicamente. La angioplastía ha demostrado claramente disminuir los síntomas en pacientes con escasa respuesta al tratamiento médico, pero no existe evidencia que muestre que comparado con el tratamiento médico disminuyan ni la mortalidad ni los eventos coronarios.

\section{b) Cirugía de puente coronario (by pass)}

La cirugía de revascularización miocárdica (CRM) consiste en la colocación de puentes sobre las arterias coronarias. Los puentes pueden ser venosos o arteriales que son más resistentes, utilizando la mamaria interna. Avances en la experiencia de las técnicas quirúrgicas, un adecuado manejo en la recuperación postoperatoria y procedimientos más modernos de circulación extracorpórea han permitido reducir la mortalidad de la cirugía de puente coronario que en los centros más importantes del mundo oscila entre el 1 y el $2 \%$. La utilización de la arteria mamaria interna aumentó mucho la vida de los puentes coronarios ya que esta arteria no desarrolla enfermedad arteriosclerótica y a los 10 años sólo se ocluye el 10\%; esto otorga una mejoría clínica más persistente. La disminución de la colesterolemia con estatinas también disminuye el riesgo de obstrucción de los puentes. ${ }^{23}$

\section{CRM vs. tratamiento medico}

La mejor evidencia sobre el rol de la CRM en pacientes ACE, parte de un metaanálisis* de siete estudios que comparan ambas estrategias. ${ }^{24} \mathrm{El}$ mismo muestra que en pacientes con enfermedad de tres vasos o compromiso del tronco de la coronaria izquierda (TCI), La mortalidad fue menor en aquellos randomizados a CRM. No se observó beneficio sobre la sobrevida en aquellos pacientes con lesión de uno o dos vasos y CRM, a menos que tuvieran comprometida la arteria des- cendente anterior proximal. Fuera de este tipo de lesiones la CRM no mostró beneficios sobre la sobrevida, pero si mejorar la angina.

CRM comparada con tratamiento medico en angina crónica estable

\begin{tabular}{cccl}
\hline $\begin{array}{l}\text { Numero de vasos } \\
\text { comprometidos }\end{array}$ & $\begin{array}{l}\text { Severidad de la is- } \\
\text { quemia en pruebas } \\
\text { funcionales }\end{array}$ & $\begin{array}{l}\text { Fracción de eyec- } \\
\text { ción del ventricu- } \\
\text { lo izquierdo }\end{array}$ & $\begin{array}{l}\text { Resultados so- } \\
\text { bre la sobrevida } \\
\text { de la CRM * }\end{array}$ \\
\hline 2 & Moderada & $>50 \%$ & Sin cambios \\
\hline 3 & Moderada & $>50 \%$ & Sin cambios \\
\hline 2 & Moderada a severa & $>50 \%$ & Sin cambios \\
\hline 3 & Moderada a severa & $>50 \%$ & Mejoria \\
\hline 2 & Moderada & $<50 \%$ & Sin cambios \\
\hline 3 & Moderada & $<50 \%$ & Mejoría \\
\hline 2 & Moderada a severa & $<50 \%$ & Mejoría \\
\hline 3 & Moderada a severa & $<50 \%$ & Mejoría \\
\hline
\end{tabular}

En este esquema se considera enfermedad de tres vasos al compromiso de dos vasos que incluya la descendente anterior proximal.

* La mejoría sobre la sobrevida ha sido documentada en ensayos clínicos randomizados en La CRM. La estrategia de comenzar con angioplastía en pacientes con enfermedad de tres vasos ha sido comparada con la cirugía sin encontrarse diferencias significativas.

\section{CRM vs. angioplastia}

El objetivo de los estudios es analizar si hay pacientes que no obtienen un beneficio en la sobrevida si se los trata incialmente con angioplastía.

Un metaanalisis* que incluye seis estudios de pacientes con enfermedad de múltiples vasos, así como dos estudios de pacientes con enfermedad de un vaso, no mostró diferencias entre los dos grupos. ${ }^{25}$ En el grupo angioplastía, la tasa de reprocedimeintos fue 10 veces mayor en el primer año, y la prevalencia de angina significativamente mayor, aunque se equiparó a los tres años.

Un gran estudio (BARI), no observó diferencias en la sobrevida luego de cinco años de seguimiento, pero mostró una diferencia a favor de la CRM en el subgrupo de pacientes diabéticos. ${ }^{26} \mathrm{Al}$ final del estudio, el $54 \%$ de los pacientes en el grupo angioplastía requirieron de procedimientos adicionales, comparado con el $8 \%$ del grupo CRM. La calidad de vida evaluada inicialmente fue mayor en el grupo CRM, pero al final del estudio los índices fueron similares. ${ }^{27}$

\section{Resumen}

CRM para pacientes con: compromiso del TCI, enfermedad de tres vaSos, enfermedad de dos vasos que involucra la descendente anterior (D/A) proximal y disminución de la función ventricular. Recomendación Clase I, nivel de evidencia A2

CRM o angioplastía para pacientes con uno o dos vasos sin compromiso de la D/A proximal y con una prueba evocadora de isquemia de alto riesgo. Recomendación Clase I, nivel de evidencia B2

CRM o angioplastía para pacientes con enfermedad de un vaso con compromiso de la D/A proximal. Recomendación Clase IIa, nivel de evidencia B2.

CRM o angioplastía para pacientes con uno o dos vasos sin compromiso de la D/A proximal que no recibieron medicación adecuada y tiene una prueba evocadora de isquemia de bajo riesgo. Recomendación Clase III, nivel de evidencia C2

El primer paso para la elección del régimen terapéutico se basa en la valoración del riesgo explicada de manera amplia y profunda en la primera parte de esta guía de manejo. El tratamiento médico se utiliza cuando este puede controlar adecuadamente la angina y no existen indicios en las pruebas funcionales que evidencien la presencia de isquemia importante. Los procedimientos de revascularización están indicados cuando la angina no se logra controlar adecuadamente con el tratamiento médico y existen habitualmente evidencias de mayor isquemia en las pruebas funcionales. En este grupo la CCG muestra habitualmente lesiones revascularizables. Si los pacientes no tienen un 
monto de isquemia muy importante pero la angina limita la calidad de vida, los procedimientos de revascularización en estos casos logran mejorar significativamente la angina pero no mejoran la sobrevida. En estos casos puede indicarse angioplastía (en ausencia de lesión de TCI), en enfermedad de tres vasos se puede indicar angioplastía o ci- rugía. La CRM ha demostrado una reducción de la mortalidad en pacientes con lesiones especiales: Compromiso del tronco de la coronara izquierda, enfermedad de tres vasos con disminución de la función ventricular, enfermedad de dos vasos con compromiso de la descendente anterior proximal.

\section{Algoritmo}

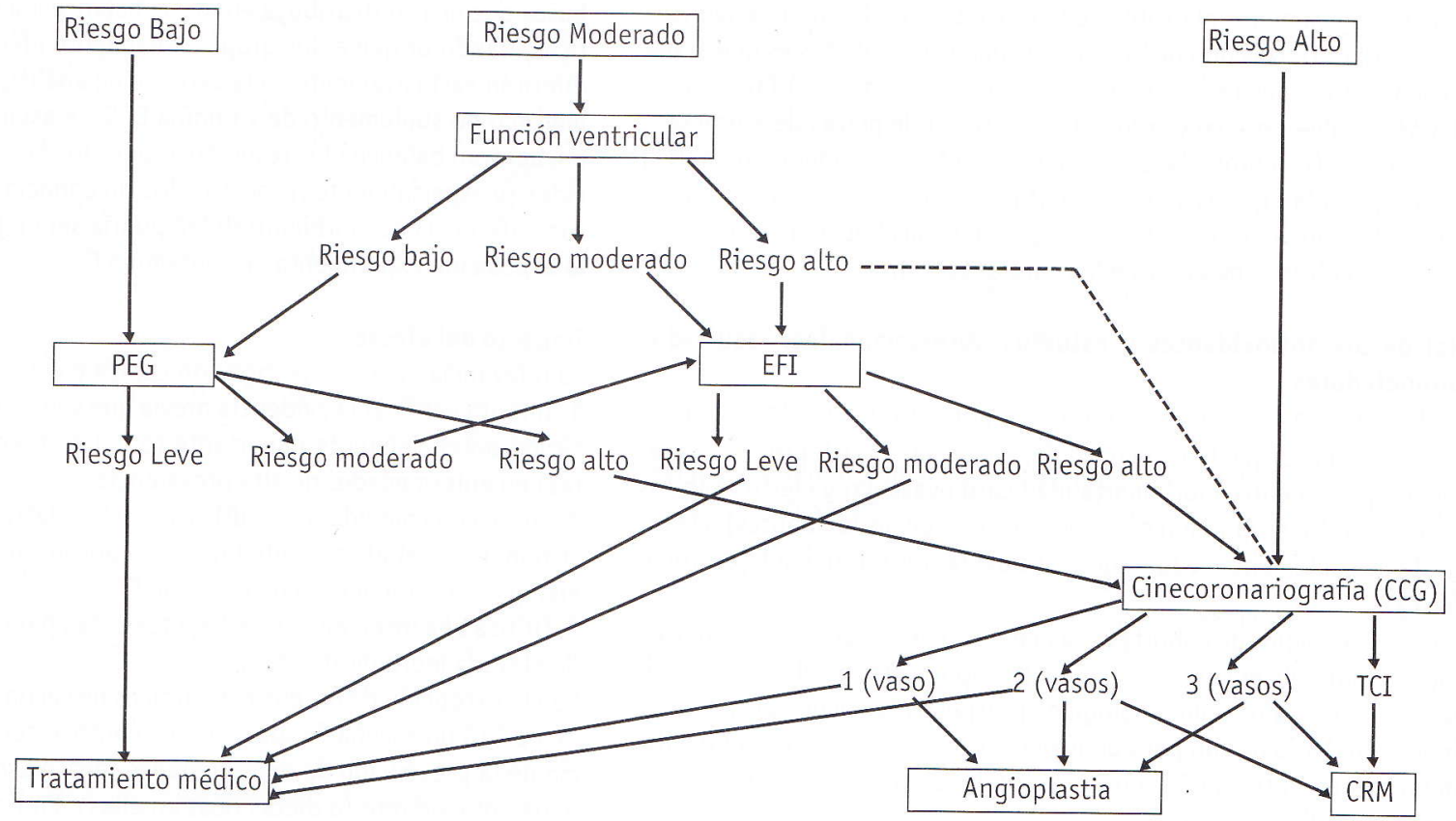

PEG : Ergometria. EFI : Evaluación Funcional por imágenes.

Para la evaluación del riesgo clínico y de las pruebas funcionales ver la primera parte de la guía de manejo.

*Ver glosario

Dr. Augusto Granel

Dr. Pablo Garcia Marletti

\section{Bibliografía}

1. Braunwald E; Heart Disease, a Textbook of Cardiovascular Medicine, 4 th. Edition 1992

2. A report of the American Heart Association/ American College of Cardiology, of the Cardiology Task Force on practice guidelinnes (comitee on management of patients with chronic stable angina). (irculation, $1999 ; 99,2829-2848$

3. Mehta R, Eagle K. Secondary prevention in acute myocardial infarction. BMJ $1998 ; 316: 838-42$

4. He J, Vupputi S, Allen K, et al. Passive smoking and the risk of coronary heart disease. A metaanalisis of epidemiological studies. N Engl J Med $1999 ; 340$ (12) :920.

5. Scandinavian Simvastatin Survival Study Group. Randomized trial of cholesterol lowering in 4444 patients with coronary artery disease : the Scandinavian simvastatin survival study (4S). Lancet $1994: 334$ (45), 1383-9

6. Sacks FM, Pfeffer LA, Moye MA, et al. The effect of pravastatin on coronary events after myocardial infarction in patients with average cholesterol levels. N. Engl. J. Med.1996; 335 :1001-1009

7. The long term intervention with pravastatin in ischeamic heart disease (LPID) study group. Prevention of cardiovascular events and death with pravastatin in patients with coronary heart disease and a broad range of cholesterol levels. N Engl J Med 1998; $339: 1349-57$

8. Bloomfield H, Robbins J, Collins P, et al. Gemfibrozil for the secondary prevention of coronary heart disease in men with low levels of HDL cholesterol. N Engl J Med 1999: 341 :410-8 9. Antiplatelet Trialist Collaboration. Collaborative review of randomized trials. of antiplatelet therapy: prevention of death, myocardial infarction and stroke by prolonged antiplatelet therapy in various categories of patients. BMJ $1994 ; 308: 81-106$

10. Swedish Angina Pectoris Aspirin Trial Group. Double blind trial of aspirin in primary prevention of myocardial infarction in patients with stable coronary artery disease. Lancet 1992 ; $340: 1421-1425$

11. Bennet CL, Lowens JM, Carvile JM. Thrombotic thrombocitopenia purpura associated with clopidogrel. N Engl J Med 2000; $342(29): 1773$

12. Ball S, Hall A. Who should be treated with angiotensin-converting enzyme inhibitors after myocardail infarction? Am. Heart. J. $1996 ; 132: 244-50$

13. Miller D. Secondary prevention for ischemic heart disease. Relative numbers needed to treat with different therapies. Arch. Intern. Med. 1997; $157: 2045-2052$ )

14. Hulley S, Grady D, Bush T, et al. Heart and estrogen/progestin repalcement study (HERS) Research group.randomized trial of estrogen plus progestin for secondary prevention of coronary heart disease in post menopausal women. JAMA $1998 ; 280: 605-613$

15. Evidence-Based, Cost-effective Risk Stratification and Management after Myocadial Infarction. Arch Intern

Med. 1997; $157:$ 273-280

16. Carbajal E, Prakash D. Coronary Heart Disease. Medical Clinics of North America 75 : 1063-1083, 1995

17. Yusuf S, Friedman I, et al. Overview of results of randomized trials in heart disease : treatment following myocardial infarction. JAMA $1988 ; 310: 830-6$

18. Solomon A, Gersh B. Management of chronic stable angina : medical therapy, percutaneous transluminal coronary angioplasty and coronary artery bypass graft surgery. Lessons from randomized trials. Ann. Intern. Med. $1998 ; 118: 216-223$

19. Furberg CD, Psaty BM, Meyer JB. Nifedipine. Dose related increase in mortality in patients with coronary artery disease. Circulation 1995; 92: 1326-31

20. Multicenter Diltiazem Postinfarction Trial Research Group. The effect of diltiazem on mortality and reinfarction after myocardial infarction. N Engl J Med 1988; 319 :385-392

21. Cohn F, Peter. Concomitant use of nitrates, calcium channel blockers and betablockers for optimal antianginal therapy. Clin. Cardiol. 1994; 17:415-421

22. Parisi AF, Folland ED, Hartigan P. A comparison of angioplasty with medical therapy in the treatment of single vessel coronary artery disease. N. Engl. J. Med., $1992 ; 326: 10-16$

23. RTA-2 Trial Participants. Coronary angioplasty versus medical therapy for angina : the second randomized intervention treatment of angina. Lancet $1997: 350 ; 461-468$

24. Yusuf S., et al. Effect of coronary by pass graft surgery on survival : overview of 10-year results from randomized trials. Lancet $1994 ; 344: 563-570$

25. Pocock SJ, Henderson RA, Richards AF, et al. Meta-analysis of randomized trials comparing coronary angioplasty with by pass surgery. Lancet $1995 ; 346: 1184-9$

26. The by pass angioplasty revascularization investigation (BARI) investigators. Comparison of CABG with PTCA in patients with multivessel disease. N. Engl. J. Med. 1996 ; $335: 217-25$

27. Hlatky MA, Rogers WJ, Johnstone I, et al. Medical care costs and qua lity of life after randomization to coronary angioplasty or coronary bypass surgery. Bypass Angioplasty Revascularization Investigation (BARI). N Engl J Med 1997;336:92-9 